\title{
Manual Method for Measuring The External Quantum Efficiency for solar cells
}

\author{
SLAMI Abdelhadi ${ }^{1 *}$, BENRAMDANE Noureddine ${ }^{2}$ \\ ${ }^{1}$ University of Abu Bakr Belkaïd, Faculty of sciences, Physic's department Research Unite « Material's Renewable Energy », \\ URMER,Fg Pasteur, BP 119, Tlemcen, 13000, Algeria \\ ${ }^{2}$ University of Djillali Liabes, Faculty of Technology Laboratory of Compenent And Materials Development, LECM , Sidi Bel-Abbes, \\ 22000, Algeria
}

\begin{abstract}
Nowadays, the research related to the solar cells is oriented to the solar cell's quantum efficiency (QE) or the Incident Photon to Charge Carrier Efficiency (IPCE) development. The determination of the external quantum efficiency (EQE) is fundamental to photovoltaic research [10]. This article proposes a fast conventional method to determine the external quantum efficiency (EQE) of a solar cell using a measuring bench (IPCE), such as the instruments and the measuring principle. The foundations necessary to study the important parts of EQE are built. The main equations and guiding principles of this measurement are reviewed.
\end{abstract}

\section{INTRODUCTION}

The photovoltaic solar energy is an important tool to reduce the cost of electricity distribution in some regions. The solar cell is considered as an essential device. It has the ability to absorb light and deliver some of the energy from the absorbed photons to the electric current carriers (electrons and holes), through the physical phenomenon called photovoltaic effect. The most important parameters those describe the performance of a solar cell are irradiance and temperature [1-2-3]. Quantum Efficiency (QE) is one of the key parameters of solar cells. It quantifies the efficiency of the conversion of light into electrons as a function of the wavelength of the incident light. The external quantum efficiency (EQE) is the fraction of incident photons on the solar cell that create electronhole pairs in the absorber. These successfully collected photons are measured over different wavelength of light. Because it is directly related to the short-circuit current of the solar cell, it is an excellent diagnostic tool to improve efficiency. This measurement is used to test structures and cellular materials, as well as to verify the reproducible production of solar cells and modules. [4]. Several authors [5-8] evaluate performance of solar cell through spectral response the dependence of the collected charge carriers on the incident photons of different wavelengths [9] or internal quantum efficiency.

In this work, we present results of external quantum efficiency (EQE) of a silicon solar cell. Measurements are carried by the Incident Photon to Charge Carrier Efficiency (IPCE) bench

The introduction should briefly place the study in a broad context and highlight why it is important. It should define the purpose of the work and its significance. The current state of the research field should be reviewed carefully and key publications cited. Please highlight controversial and diverging hypotheses when necessary. Finally, briefly mention the main aim of the work and highlight the principal conclusions. As far as possible, please keep the introduction comprehensible to scientists outside your particular field of research. References should be numbered in order of appearance and indicated by a numeral or numerals in square brackets, e.g., [1] or [2,3], or [4-6]. See the end of the document for further details on references.

\section{Quantum efficiency}

The quantum efficiency of solar cell is the ratio of the numbers of carriers collected by the solar cell to the number of incident photons. It describes the response of the device with different wavelengths of light. It represents the ratio of the number of collected carriers in short-circuits operating condition by the number of incident photons for a given wavelength. Its expression is given by equation 1 [10]:

$\operatorname{EQE}\left(\lambda, r, S_{g b}\right)=\frac{\text { h.c. } J_{S C}}{q \cdot \lambda \cdot I_{0}(\lambda)}$

Where $\mathrm{h}$ is Planck's constant, $\mathrm{q}$ is the charge of electron, $\mathrm{c}$ is the velocity of light, Jsc is the short-circuit current, $\lambda$ is the wavelength of exciter light and $\operatorname{IO}(\lambda)$ the electric current due to the photoelectric effect.

The internal quantum efficiency represents the ratio of short-circuit photocurrent density by the number of photons that have really been absorbed into the cell. Its expression is given by 2 [7]:

$$
\operatorname{IQE}(\lambda)=\frac{E Q E}{q \cdot \lambda \cdot I_{0}(\lambda)}
$$

\section{Experimental method}

There are few turnkey quantum efficiency measurement systems available. This paragraph presents a configuration on the hardware needed for $\mathrm{QE}$ measurement, from the generation of the beam of light to its absorption by the cell under test. Measurements of the external quantum efficiency can be made using a measuring bench (IPCE) connected to a computer (Figure 1).

*Corresponding author: slamiabdelhadi@gmail.com 


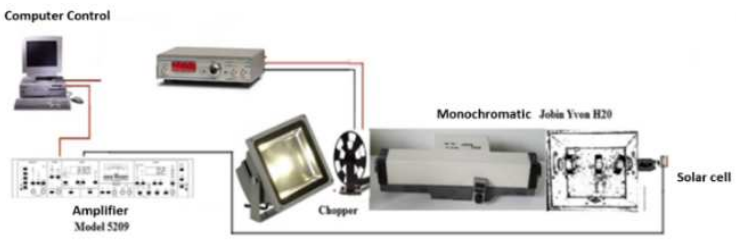

Figure 1. Measuring bench (IPCE) for measurements of the external quantum efficiency $(\mathrm{QE})$.

In the measuring bench, a reference photodiode is initially mounted in place of the photovoltaic cell to be tested. This photodiode is used to record the intensity of the incident light beam and the emission spectrum of the lamp. These two quantities can indeed vary significantly over time. The first reference signal is therefore "backround" from the spectrum of action. The reference is used to draw the photovoltaic spectrum of the solar cell. The extracted signals (from the photodiode and the photovoltaic cell) are voltage and current respectively. This photodiode is used as a reference to record the intensity of the incident light beam and the emission spectrum of the lamp. These two quantities can indeed vary significantly over time depending on the wear of the lamp. The first reference signal is therefore a "backround" From our spectrum of action, in terms of spectroscopy. This reference is used to draw the photovoltaic spectrum of the solar cell, it can be noted in this figure that the signals extracted from the reference photodiode and the photovoltaic cell are voltage and current respectively.

In practice this current is amplified and converted into voltage. The signal from the photovoltaic cell is often sent directly to the input $\ll$ current $\gg$ of the synchronous detection. We can then divide this montage into two parts:

The optical part (light source, modulator and light monochromator), a monochromator selects the length of the light beam of the halogen lamp. The selection of the wavelengths is ensured between 350 and $850 \mathrm{~nm}$. The light signal is then modulated by a beam chopper whose frequency will be chosen as the reference of the synchronous detection. A set of mirrors allows directing the beam on the sample. And the electronic part (currentvoltage conversion amplifier of the reference photodiode and synchronous detection).

\section{Results and discussion}

\subsection{Preparation of solar cells.}

To prepare our measuring cells, we used broken photovoltaic solar cell fragments of monocrystalline type. For ease of handling we adapted the cell support to the diameter of the sample chamber (Figure 02).

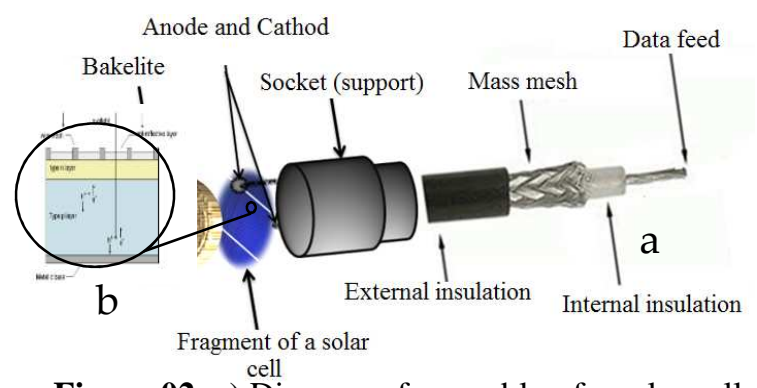

Figure 02: a) Diagram of assembly of a solar cell. b) Schematic representation of a conventional solar cell.It represents the creation of electron-hole pair eand $\mathrm{h}+$, respectively [8]

\subsection{Qualitative analysis of the results}

\subsubsection{Definition of the numbers of the electrons.}

On the one hand, and at first, we excited the sample of a solar cell with an area of 2.10-4 m2 which is charged by a resistance of $30 \mathrm{~K} \Omega$.

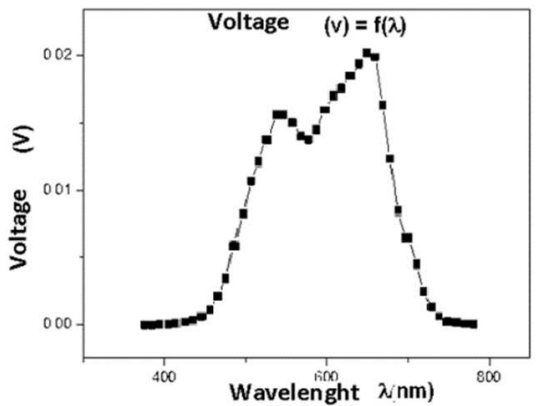

Figure 03. Voltage of monocristalline solar cell.

The sample photon current is converted into voltage by an I-V converter. This voltage is filtered by the synchronous detection, then measured by a multimeter and finally recorded by a computer. For each wavelength, the voltage delivered at the output of the string with the sample is first measured, then in a second time the voltage with a reference whose spectral response is perfectly known throughout the spectrum [01] [03] [04] [06] [07].

With a light excitation source (250w tungsten lamp) to obtain the voltage at the charging terminals as a function of the wavelengths (Figure 03). Using the ohm law to calculate the current (Figure 04).

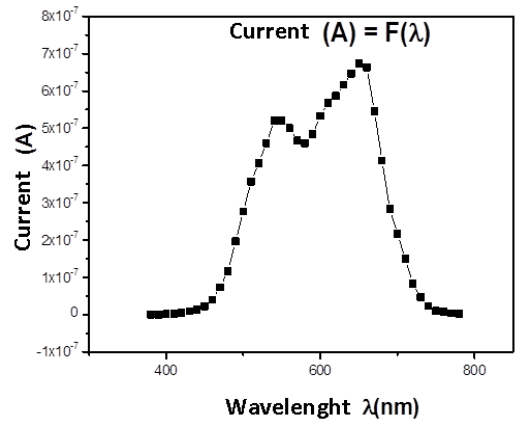

Figure 04. Current of monocristalline solar cell.

From the current, we can calculate the number of electrons per second (Figure 05) with the relation:

$$
n=\frac{A}{q}=\left[\frac{C / S}{C}\right]
$$

$\mathrm{n}$ : it is the number of electrons.

A: it's the current [coulomb per second].

$\mathrm{q}:$ it is the electric charge [coulomb] (1.6.10-19c). 


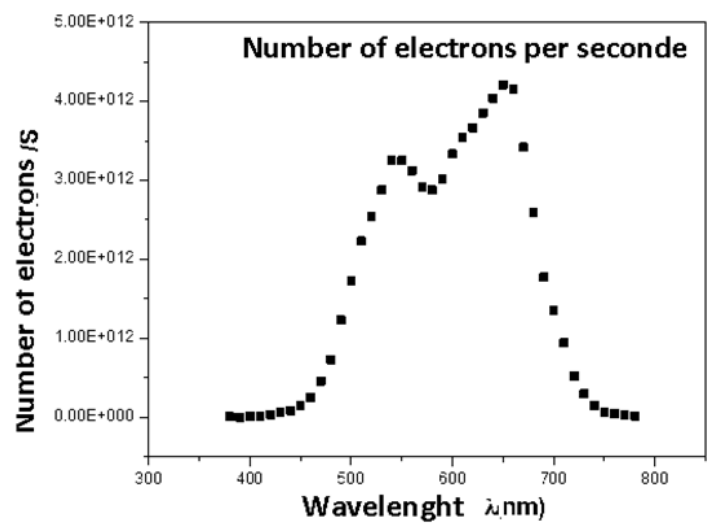

Figure 05. Number of electrons per seconde of the solar cell.

\subsubsection{Definition of the numbers of the photons}

To determine the number of photons we replaced the solar cell with a light meter, we were able to record the illumination received by the luxmeter and the wavelength (Figure 06).

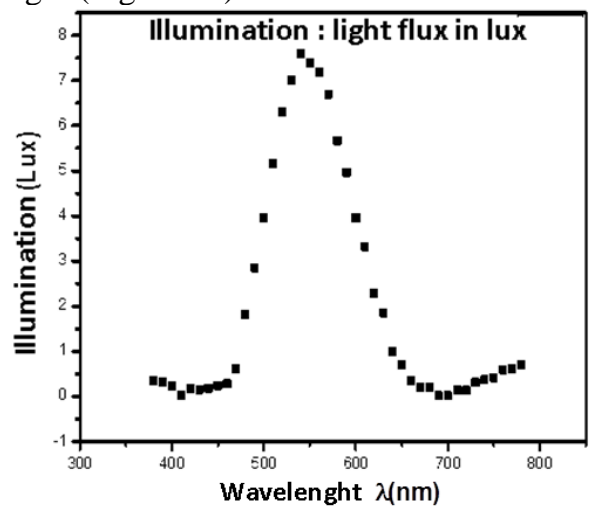

Figure 06. The lux illumination received by the solar cell.

The illumination must be converted into a luminous flux which represents the energy transported by the beam (Figure 07) by the relation (4):

$$
E=\frac{\Phi}{S}
$$

With:

$\Phi$ : Luminous flux, in lumens ( $1 \mathrm{~m})$.

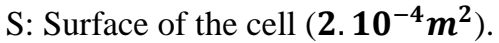

$\mathrm{E}$ : Illuminance, in lux (lx).

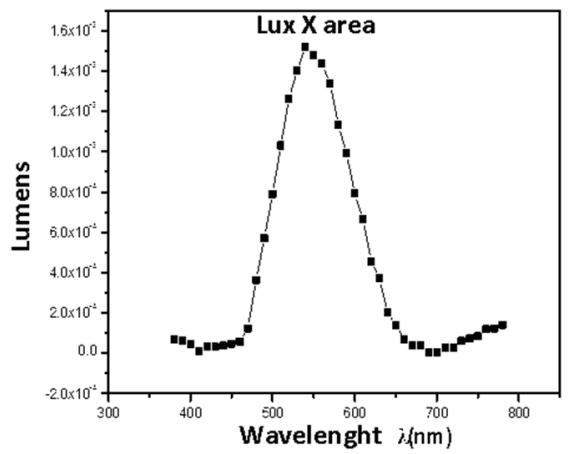

Figure 07. Luminous flux in lumens received by the solar cell.
Then we present the curve of the luminous efficiency photo pic of the human eye (Figure 08). Which will allow us to convert lumens into watts.

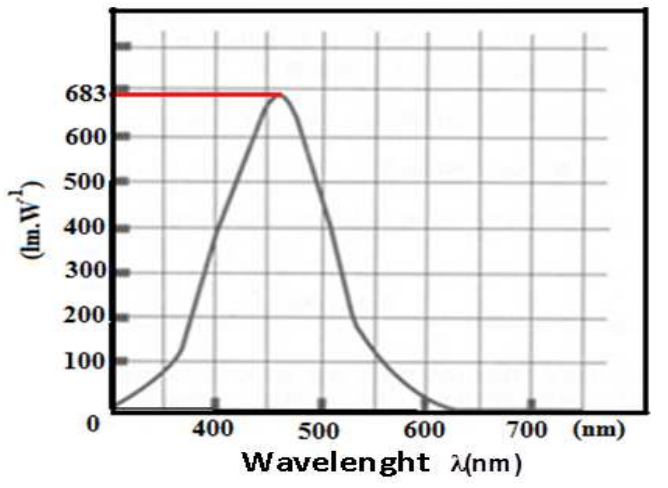

Figure 08. The photopic luminous efficiency of the human eye.

We did the normalization compared to the max (Fig. 09), to achieve proportionate results.

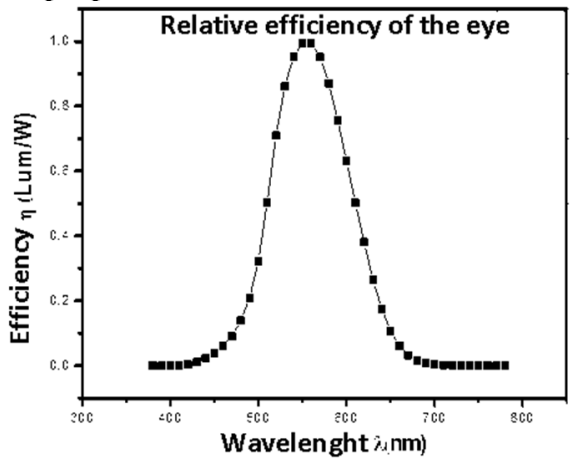

Figure 09. Relative efficiency of normalized human eye compared to max.

After, we compared the luminous flux in lumens with the relative efficiency of the normalized eye to find the power received by the luxmeter (Figure 10).

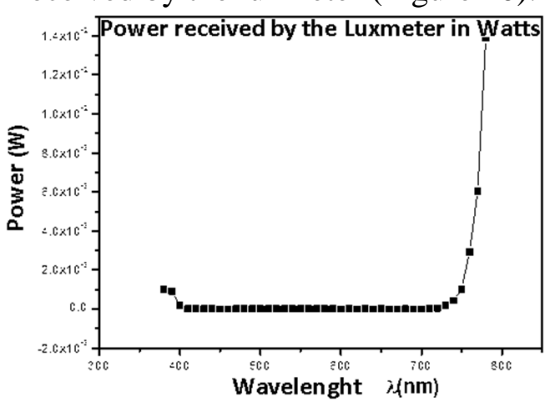

Figure 10. The power received by the luxmeter in Watt.

In a second time and after these results we calculated the energy of the photons in Joule (Figure 11) with the relation:

$$
E_{J}=\frac{1,24}{\lambda(\mu m)} \cdot 1,6 \cdot 10^{-19}
$$




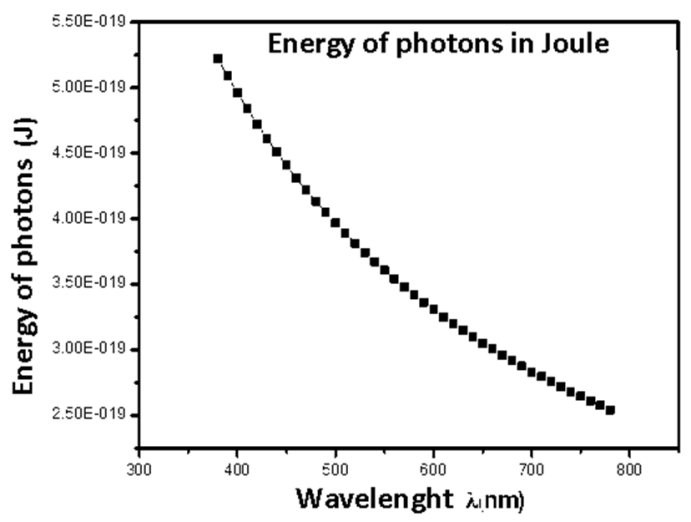

Figure 11. Energy of the photons in Joule. Finally, we have the number of photons per second (Figure12) with:

$$
N b r=\frac{E(W / S)}{P(W)}
$$

Nbr : Number of photons per seconde. E :Energy of the photons in Joule.

$\mathrm{P}$ : Power received by the luxmeter in watts.

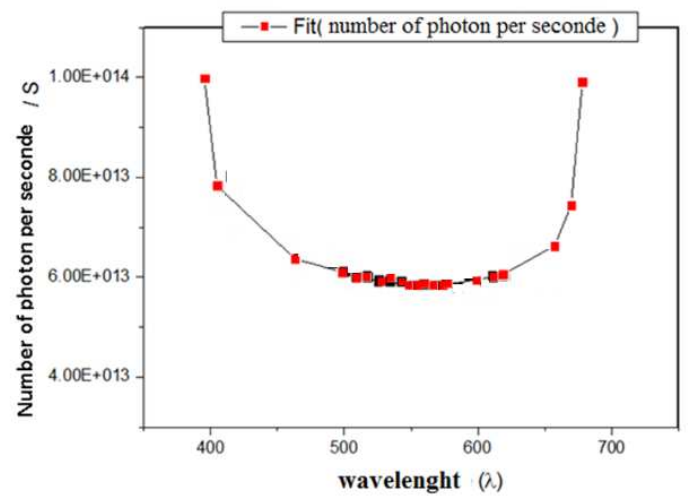

Figure 12. Numbers of photons per seconde.

Except for the edges of the curve, the dispersion may be due to measurement errors and the low sensitivity of luxmeter, there is a decrease in the photometric flux for low energies.

Finally, we divide the number of electrons on the number of photons to obtain the external quantum efficiency (Figure 13).

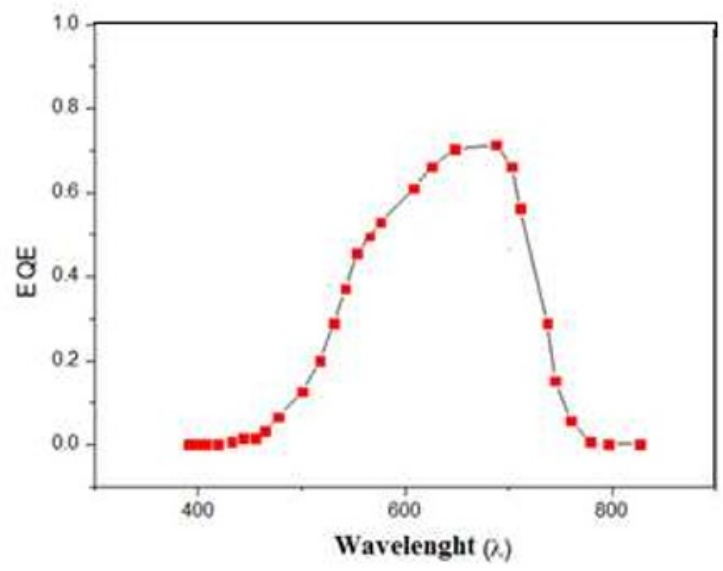

Figure 13. External quantum efficiency.
The external quantum efficiency gives the probability that photons will be absorbed to generate electron-hole pairs. A maximum in IPCE of $75 \%$ is reached at a wavelength near $693 \mathrm{~nm}$.

The ideal of external quantum efficiency must be rectangular however due to reality, this number is not rectangular and also determines the value of the photocurrent produced by the cell:

$J_{S C}=e \int_{\lambda \min }^{\lambda \max \int} E Q E(\lambda) \cdot \Phi(\lambda) \cdot d \lambda$

This expression shows that the higher the EQE, the stronger the current.

\section{Conclusion}

A conventional EQE measurement system cannot be used in an industrial production environment. This means that the measurement time scales with the number of points (wavelengths) measured and more time is needed for those points in the spectrum where the solar cell has a weak response [7] [8] [9]. In practice, the conversion of light energy into electrical energy is not total. Different losses influence the quantum efficiency of a cell. In most cases they are due to the nature of the material and the technology used. All photons having a wavelength greater than that associated with the gap of the semiconductor can not generate an electron-hole pair, and are therefore lost. The efficiency of a cell also depends on the number of photons penetrating it. In this work, results showed that external quantum efficiency is found to be increase with wavelength reached to maximum of $693 \mathrm{~nm}$. It is due to an increase of carriers collection. However, beyond this, it decreased rapidly owing to the effect of recombination and optical losses due to transmission and the reflection phenomena.

\section{References}

1. M. Green, "Solar Cells: Operating Principles, Technology and System Applications". Englewood Cliffs, NJ: Prentice-Hall, (1992).

2. L. Zeng, M.Li, Y. Chen and H. Shen, "A simplified method to modulate colors on industrial multicrystalline silicon solar cells with reduced current losses", Solar Energy 103, (2014).

3. S..K. Sharma, H. Im, D.Y. Kim and R.M. Mehra, "Review on Se and S- doped hydrogenated amorphous silicon thin films", Indian Journal of Pure \& Applied Physics, 52, (2015).

4. M. Green, "Solar Cells: Operating Principles, Technology and System Applications". Englewood Cliffs, NJ: Prentice-Hall, (1992).

5. R. Antony, "Optoelectronic realization and characterization of electro-luminescent diodes based on electroactive polymers deposited with the assistance of an ion beam", Thesis, Limoges (1998).

6. A.l Cardona A.J., P.Chica C.A., D.H. Ospina Barragán, "Method for Calculating Quantum Efficiency and Spectral Response of Solar Cells Using LabVIEW.In:Building-Integrated 
Photovoltaic Systems (BIPVS)" .Springer $\operatorname{book}(2018)$,

7. L.J. Geerligs, P. Manshanden, I. Solheim, E.J. Ovrelid, A.N. Waernes, "Impact of Common Metallurgical Impurities on mc-Si Solar Cell Efficiency", 21st European Photovoltaic Solar Energy Conference and Exhibition, 4-8 September, Dresden, Germany (2006).

8. A.Diouf, M. Savadogo, S. Mbodji, "External Quantum Efficiency (EQE) and Internal Quantum Efficiency (IQE) in a 3D Cylindrical Modeling Study", Journal of Scientific and Engineering Research, 5 (2018).

9. H. Mäckel, W. Warta and A. Cuevas, "Spectral response of the photoconductance: a new technique for solar cell characterization", Solar World Congress (2001).

10. A. Luque, S. Hegedus, "Handbook of Photovoltaic Science and Engineering", Wiley (2011). 